\section{HIV-1 error revealed}

SIR - We regret to inform Nature's readers that one experimental result in our recent Letter ${ }^{1}$ is incorrect. The clones of one of the HIV-1 (human immunodeficiency virus type 1) mutants that we studied, called mutant 4 , had unintended mutations in addition to the intended mutations in reverse transcriptase codons $74,103,215$ and 219. We discovered these unintended mutations in reverse transcriptase only on further sequencing; they can explain why viruses derived from these clones were not viable. This explains the discrepancy between our report ${ }^{1}$ and the findings of Larder et al. ${ }^{2}$ and Emini et al. (see below). We believe that all other data in our report are correct, and we have confirmed the in vitro treatment results in subsequent experiments ${ }^{3}$.

We had hypothesized that one rationale for the use of several drugs directed against the same HIV-1 target was that some potential paths to multidrug resistance might not be available to the virus and never be seen under multidrug selection pressure. However, some mutations that confer resistance to non-nucleoside reverse transcriptase inhibitors can combine with AZT and ddI (2',3'dideoxyinosine) resistance mutations to yield viable, triply resistant viruses in vitro. In extensions of the in vitro drug selection experiments we mentioned in our Letter ${ }^{4}$, we have also been able to select for triply resistant viruses similar to those described by Larder et al. ${ }^{2}$.

Interactions between drug-resistance mutations will help to explain the structure and function of reverse transcriptase. However, caution is necessary in extrapolating from mutational interactions noted in vitro to the clinical potential of a particular combination. The primary criterion for choosing drugs to combine in clinical trials should be antiviral effectiveness. Our in vitro treatment experiments ${ }^{1,3}$ and clinical-trial data ${ }^{5}$ suggest that the combination of nucleoside and non-nucleoside reverse transcriptase inhibitors may provide prolonged antiviral effectiveness. Clinical trials comparing AZT, ddI and a non-nucleoside reverse transcriptase inhibitor to more standard combinations (such as AZT and ddI or $\mathrm{AZT}$ and $\mathrm{ddC}\left(2^{\prime}, 3^{\prime}\right.$-dideoxycytidine $\left.)\right)$ are now under way.

We agree with Emini et al. that multidrug-resistant variants are likely to be selected eventually during combination

1. Chow, Y. K. et al. Nature 361, 650-654 (1993)

2. Larder, B. A. Kellam. P. \& Kemp, S. D. Nature (in the press)

3. Mazzulli, T., Chow, Y.-K., Merrill, D. P. \& Hirsch, M. S. Abstract PO-B26-2082 IX Int, AIDS Conf, Berlin (1993).

4. Chow, Y.-K., Merrill, D. P. Kaplan, J. C., D'Aquila, R. T. \& Hirsch, M. S Abstract WS-A19-6IXInt. AlDS Conf. Berlin (1993)

5. Havlir, D. et al. Abstract WS-B26-1 IX Int. AIDS Conf. Berlin (1993) therapy. But it remains important to explore new strategies for available drugs. Some drug combinations, particularly those which are more potent inhibitors of HIV-1 replication, may prolong the benefit of therapy more than others. This can only be determined by clinical trials.

Yung-Kang Chow

Martin S. Hirsch

Joan C. Kaplan

Richard T. D'Aquila

Infectious Disease Unit,

Massachusetts General Hospital,

Boston,

Massachusetts 02114, USA

\section{HIV and multidrug resistance}

SIR - The reverse transcriptase of human immunodeficiency virus type 1 (HIV-1) is inhibited by the nucleoside analogues AZT and $2^{\prime}, 3^{\prime}$-dideoxyinosine (ddI) as well as by the non-nucleoside compounds BI-RG-587 and L-697,661 (refs 1, 2). Unfortunately, the usefulness of these inhibitors as single therapeutic agents is limited by the emergence, in treated in- series of mutant HIV-1 variants. The variants are listed in the table, together with their respective susceptibilities to the four test inhibitors. The combined effects of the expressed substitutions on virus susceptibility, particularly to AZT, are complex. For instance, we confirmed a previous observation that substitution at residue 74 resensitizes virus to $A Z T$ in the presence of the AZT resistanceengendering mutation at residue 215 (ref. 7 ). But the effect of the residue 74 alteration was abrogated in some cases (variants $B$ and $C$ but not $E$ ) by co-expression of the non-nucleoside inhibitor-specific substitutions at residues 103 or 106 .

In any case, variants $B$ and $C$ were multiply resistant to AZT, ddI, L-697,661 and/or BI-RG-587, whereas variant $D$ was resistant to ddI and both non-nucleoside inhibitors. Surprisingly, variant $\mathrm{E}$ is the identical putative nonviable variant constructed by Chow et al. ${ }^{6}$. In our hands, this mutant virus exhibited growth kinetics similar to wild-type virus (data not shown) and expressed a multiply resistant phenotype. We do not know why our observation differs from that of Chow et al. Nonetheless, we have demonstrated that multiply resistant viable HIV-1 variants can exist. The apparent phenotypic interactions that occur among co-expressed reverse transcriptase mutations reflect

\begin{tabular}{|c|c|c|c|c|c|}
\hline \multicolumn{6}{|c|}{ SUSCEPTIBILITY OF HIV-1 VARIANTS TO TEST INHIBITORS } \\
\hline \multirow[t]{2}{*}{ Variant } & \multirow[t]{2}{*}{ Amino-acid substitutions } & \multicolumn{4}{|c|}{$\mathrm{IC}_{95}(\mu \mathrm{M})$} \\
\hline & & AZT & ddl & BI-RG-587 & L-697, 661 \\
\hline Wild type & None & 0.025 & 12.5 & 0.400 & 0.100 \\
\hline A & $74(\mathrm{~L} \rightarrow \mathrm{V}), 215(\mathrm{~T} \rightarrow \mathrm{Y})$ & 0.100 & 100.0 & 0.100 & 0.025 \\
\hline B & $74(\mathrm{~L} \rightarrow \mathrm{V}), 103(\mathrm{~K} \rightarrow \mathrm{N}), 215(\mathrm{~T} \rightarrow \mathrm{Y})$ & 50.0 & 100.0 & $>3.0$ & 0.800 \\
\hline C & $74(\mathrm{~L} \rightarrow \mathrm{V}), 106(\mathrm{~V} \rightarrow \mathrm{A}), 215(\mathrm{~T} \rightarrow \mathrm{Y})$ & 50.0 & 100.0 & $>3.0$ & 0.100 \\
\hline D & $74(\mathrm{~L} \rightarrow \mathrm{V}), 181(\mathrm{Y} \rightarrow \mathrm{C}), 215(\mathrm{~T} \rightarrow \mathrm{Y})$ & 0.100 & 100.0 & $>3.0$ & $>3.0$ \\
\hline$E$ & $74(\mathrm{~L} \rightarrow \mathrm{V}), 103(\mathrm{~K} \rightarrow \mathrm{N}), 215(\mathrm{~T} \rightarrow \mathrm{Y}), 219(\mathrm{~K} \rightarrow \mathrm{Q})$ & 0.100 & 100.0 & $>3.0$ & 0.800 \\
\hline
\end{tabular}

Variant proviruses were constructed and 95 per cent virus inhibitory concentrations were determined as described in ref. 3.

fected persons, of resistant virus variants (see refs 3-5). Chow et al. ${ }^{6}$ have proposed the concept of "convergent combination therapy", that simultaneous multiple inhibitor therapy directed against the HIV-1 reverse transcriptase would delay the emergence of multiply resistant virus strains.

Given the contrived nature of the cellculture experiments of Chow et al ${ }^{6} \mathrm{de}$ signed to select resistant virus, we decided to determine directly if multiply resistant virus could indeed exist by constructing a

\section{Scientific Correspondence}

Scientific Correspondence is intended to provide a forum in which readers may raise points of a scientific character. They need not arise out of anything published in Nature. In any case, priority will be given to letters of fewer than 500 words and five references. significant structural and functional flexibility by the enzyme. This flexibility permits the expression of virus variants that can be resistant to various combinations of reverse transcriptase inhibitors. Given the large quantities of virus seemingly present in infected individuals ${ }^{8}$, such variants are likely to be selected during multiple therapy.

Emilio A. Eminl, Donald J. Graham, Leah Gotlib, Jon H. Condra, Vera W. Byrnes, William A. Schlelf Merck Research Laboratories, West Point, Pennsylvania 19486, USA

1. Goldman, M.E. etal. Proc. natn. Acad. Sci.U.S.A. 88 6863-6867 (1991)

. Merluzzi, V.J.et al. Science 250, 1411-1413 (1990)

3. Byrnes, V. W. et al. Antimicrob. Ag. Chemother. (in the press).

Richman D etal Proc natn Acad Sci US A 88 11241-11245(1991)

5. Nunberg. J.H. et al. J. Virol. 65, 4887-4892 (1991) 6. Chow, Y.-K. et al. Nature 361, 650-654 (1993). 7. St Clair, M. H. etal. Science 253, 1557-1559 (1991) 8. Piatak. M. etal. Science 259, 1749-1754 (1993). 